\title{
Decidual Cox2 inhibition improves fetal and maternal outcomes in a preeclampsia-like mouse model
}

\author{
Jenny L. Sones, ${ }^{1,2}$ Jeeyeon Cha, ${ }^{3}$ Ashley K. Woods, ${ }^{1}$ Amanda Bartos, ${ }^{3}$ Christa Y. Heyward, ${ }^{1}$ \\ Heinrich E. Lob, ${ }^{1}$ Catherine E. Isroff, ${ }^{1}$ Scott D. Butler, ${ }^{1}$ Stephanie E. Shapiro, ${ }^{1}$ Sudhansu K. Dey, ${ }^{3}$ \\ and Robin L. Davisson ${ }^{1,4}$ \\ 'Biomedical Sciences, College of Veterinary Medicine, Cornell University, Ithaca, New York, USA. ${ }^{2}$ Veterinary Clinical \\ Sciences, School of Veterinary Medicine, Louisiana State University, Baton Rouge, Louisiana, USA. ${ }^{3}$ Division of \\ Reproductive Sciences, Cincinnati Children's Research Foundation, Cincinnati, Ohio, USA. ${ }^{4}$ Cell and Developmental \\ Biology, Weill Cornell Medical College, New York, New York, USA.
}

Preeclampsia (PE) is a disorder of pregnancy that manifests as late gestational maternal hypertension and proteinuria and can be life-threatening to both the mother and baby. It is believed that abnormal placentation is responsible for the cascade of events leading to the maternal syndrome. Embryo implantation is critical to establishing a healthy pregnancy. Defective implantation can cause adverse "ripple effects," leading to abnormal decidualization and placentation, retarded fetal development, and poor pregnancy outcomes, such as PE and fetal growth restriction. The precise mechanism(s) of implantation defects that lead to PE remain elusive. BPH/5 mice, which spontaneously develop the cardinal features of PE, show peri-implantation defects including upregulation of Cox2 and IL-15 at the maternal-fetal interface. This was associated with decreased decidual natural killer (dNK) cells, which have important roles in establishing placental perfusion. Interestingly, a single administration of a Cox2 inhibitor (celecoxib) during decidualization restrained Cox2 and IL-15 expression, restored dNK cell numbers, improved fetal growth, and attenuated late gestational hypertension in $\mathrm{BPH} / 5$ female mice. This study provides evidence that decidual overexpression of Cox2 and IL-15 may trigger the adverse pregnancy outcomes reflected in the preeclamptic syndrome, underscoring the idea that Cox2 inhibitor treatment is an effective strategy for the prevention of PE-associated fetal and maternal morbidity and mortality.

Authorship note: J.L. Sones and J. Cha contributed equally to this work. S.K. Dey and R.L. Davisson are co-senior authors.

Conflict of interest: The authors have declared that no conflict of interest exists.

Submitted: November 20, 2015 Accepted: February 16, 2016 Published: March 17, 2016

Reference information: JCI Insight. 2016;1(3):e75351. doi:10.1172/jici.insight.75351.

\section{Introduction}

The pathophysiology underlying preeclampsia (PE) is often described as having 2 stages, the first involving abnormal placentation characterized by poor trophoblast invasion, incomplete vascular remodeling, and placental hypoxia (1), while the second stage presents as the maternal syndrome of hypertension and proteinuria (2). While the cause and onset of these mechanisms are unclear, a proposed etiology is inadequate uterine angiogenesis and placental vasculogenesis at the time of implantation (3)

Early pregnancy events depend on tightly regulated signaling pathways to ensure pregnancy success. In rodents and humans, implantation is the first coordinated encounter between the mother and fetus $(1,2)$. Decidualization, or the differentiation of uterine stromal cells into a transient, specialized secretory decidual tissue, is required in both species to complete implantation. This process is accompanied by marked uterine angiogenesis and recruitment of immune cells to the decidua that aid in vascular remodeling to facilitate adequate uteroplacental perfusion $(1,4)$. Aberrations in implantation and decidualization processes can have "ripple effects" that may lead to poor pregnancy outcomes, including abnormal placentation, fetal loss or growth restriction, and even infertility (5). The precise role of implantation and decidualization in the pathogenesis of PE and associated adverse pregnancy outcomes, such as fetal growth restriction (FGR), has not yet been identified. Pregnancies characterized by PE have inadequate remodeling of spiral arteries within the decidua and poor placental perfusion, triggering placental hypoxia and the 
maternal syndrome (1). Since the placenta is formed in the first trimester, the placental pathologies seen in $\mathrm{PE}$ are thought to originate early in pregnancy. However, dysregulation in these processes are not easily studied at early gestational time points in women.

Critical to implantation and decidualization is the coordinated expression of vasoactive agents, cytokines, growth factors, transcription factors, and morphogens that act as autocrine, paracrine, and juxtacrine factors (6). In particular, Cox2-derived prostaglandins are crucial for implantation, decidualization, placental formation, and angiogenesis throughout pregnancy $(5,7,8)$. Expression of prostaglandin synthase 2 (Ptgs2; encoding Cox2) is first detected during pregnancy in mice in the uterine luminal epithelium and stroma around the blastocyst beginning at E4.5 with the attachment reaction (9). Its expression increases with decidualization, peaks at E7.5 in the uterine stroma, and becomes nearly undetectable by the time the placenta is formed. Defective implantation and decidualization in Ptgs2 ${ }^{-1-}$ mice has been shown to be secondary to dysregulated vascular events and VEGF signaling (10).

Coincident with decidualization in humans and mice is the appearance of decidual NK (dNK) cells, the predominant immune cell at the maternal-fetal interface (11-13). Upon stimulation by IL-15, mature dNK cells maintain decidual integrity and produce factors, such as IFN- $\gamma$ (13), that directly modify decidual vessels and promote angiogenesis, including VEGF (14). Women with PE often show signs early in pregnancy of angiogenic imbalances including aberrations in VEGF, placental growth factor (a member of the VEGF family), and the soluble VEGF receptor (sFlt), and inadequate peri-implantation angiogenesis and vascularity at the maternal-fetal interface has been proposed as a mechanism (3) but has not yet been experimentally tested. Since regulated Cox 2 expression and dNK cell presence are critical for these processes in early pregnancy, we sought to investigate their roles in the context of PE.

Our laboratory has shown that the BPH/5 strain of mice spontaneously develops a PE-like syndrome during pregnancy, including late gestational hypertension and proteinuria, which resolve upon delivery (15). BPH/5 pregnancies are further characterized by abnormal placentation and fetal loss, with approximately one third of all implantation sites resorbed by mid to late gestation $(15,16)$. This phenomenon results in smaller live litter sizes and lower birth weights in those neonatal pups that do survive (15, 16). $\mathrm{BPH} / 5$ mice also demonstrate angiogenic imbalances, as evidenced by decreased circulating and placental VEGF protein levels at early gestational time points (E9.5-E12.5) corresponding to the time of early fetal demise $(17,18)$. This suggests that the initial angiogenic insult in BPH/5 mice likely occurs early in pregnancy.

Dysregulation of peri-implantation events can alter the course of pregnancy progression and may create adverse "ripple effects" to derail pregnancy outcome. These initiating pathologies occur during a woman's first trimester, and it is extraordinarily difficult to study these events, given the logistical and ethical concerns. Since the BPH/5 mouse model lends itself to longitudinal investigation of the entire pregnancy, we tested the hypothesis that aberrations early in pregnancy incur retarded fetal and placental development as well as the maternal syndrome in this model. Here, we show that dysregulated Cox 2 and IL-15 during the peri-implantation period resulted in inferior fetal growth and placental development and reduced $\mathrm{dNK}$ cell numbers at the maternal-fetal interface. More important, early targeting of aberrant Cox 2 levels with a selective inhibitor (celecoxib) appropriately restricted Cox 2 and IL-15 and restored dNK cell numbers and improved fetal morbidity/mortality and late gestational hypertension in $\mathrm{BPH} / 5$ mothers. This is the first report, to our knowledge, of early pregnancy defects in the context of PE that point toward a pivotal and previously unrecognized role of Cox2 inhibition. These results align with those of a recent study showing that low-dose aspirin given prophylactically to high-risk women early in pregnancy is associated with reduced risk of PE and FGR (19) and may provide a basis for further studies in humans.

\section{Results}

Aberrations during the peri-implantation period characterize BPH/5 pregnancies. Defective implantation leads to adverse "ripple effects" throughout pregnancy, including abnormal placentation, impaired fetal development, and adverse pregnancy outcomes $(1,5,20)$. Since a PE-like syndrome, including both maternal and fetoplacental abnormalities, develops spontaneously in $\mathrm{BPH} / 5$ mice $(15,16)$, we examined peri-implantation events in these mice. One of the earliest signs of implantation is increased endometrial vascular permeability at the site of blastocyst attachment, which is identified by discrete blue bands along the uterine horns at the attachment sites after injecting a macromolecular blue dye solution (21). Using this method, we observed that, while embryos of the control strain (C57B16, C57) were evenly spaced along the uterine 
A

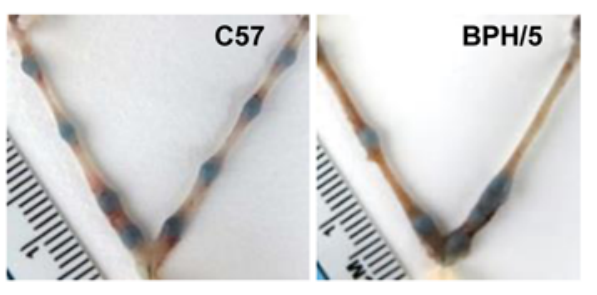

B

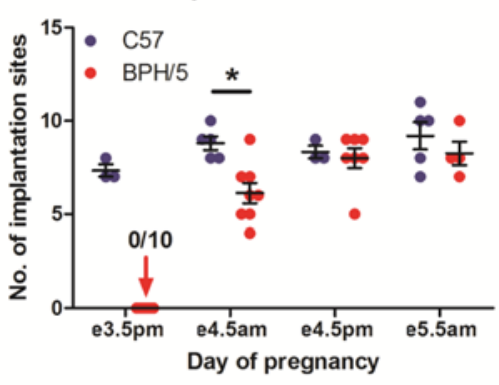

C

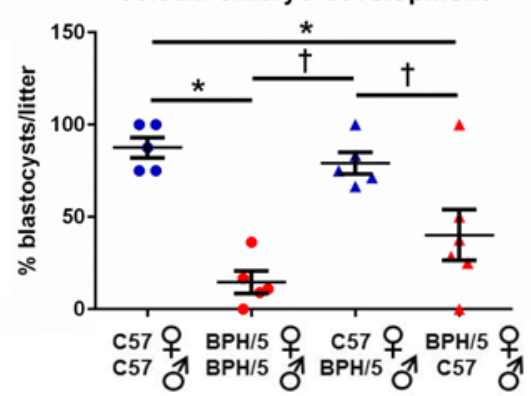

D Alkaline phosphatase staining

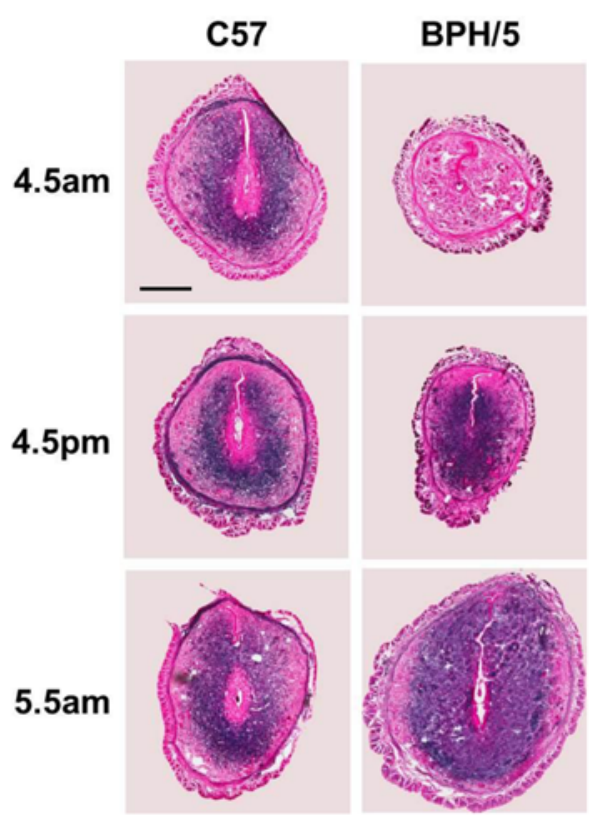

E Alkaline phosphatase quantitation

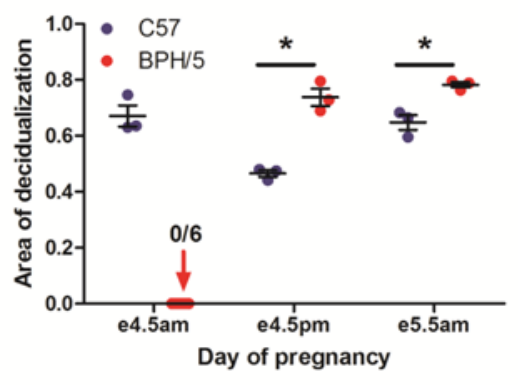

Figure 1. BPH/5 female mice exhibit dysregulated peri-implantation events. (A) Representative pontamine bluestained implantation sites from $\mathrm{C} 57$ and $\mathrm{BPH} / 5$ mice (E5.5). Area of embryo clustering is bracketed. (B) Time course of implantation sites counted per litter in pregnant $\mathrm{C} 57$ and $\mathrm{BPH} / 5$ females during the peri-implantation period. $n=$ 7-10. ${ }^{*} P<0.05$ versus gestation-matched $C 57$ females using a 2-way ANOVA. (C) Quantification of staged preimplantation embryos flushed from strain-matched $(\mathrm{C} 57 \times \mathrm{C} 57 ; \mathrm{BPH} / 5 \times \mathrm{BPH} / 5)$ and reciprocal crosses of $\mathrm{C} 57$ females $\times \mathrm{BPH} / 5$ males $(\mathrm{C} 57 \times \mathrm{BPH} / 5)$ and $\mathrm{BPH} / 5$ females $\times[57$ males $(\mathrm{BPH} / 5 \times[57) . n=4-6$ females; $40-50$ embryos. ${ }^{*} P<0.05$ versus $C 57 \times C 57$ and $\dagger P<0.05$ versus $C 57 \times B P H / 5$, by 1 -way ANOVA. (D) Representative images of ALP-stained implantation sites harvested from $\mathrm{C} 57$ and $\mathrm{BPH} / 5$ females during the peri-implantation period. Scale bar: $500 \mu \mathrm{m}$. (E) Quantification of the area of decidualization (purple staining) represented as a ratio of the total implantation site area. $n=3-6$ sections. ${ }^{*} P<0.05$ versus gestation-matched $C 57$ females, by 2 -way ANOVA. Data represent the mean \pm SEM. ALP, alkaline phosphatase.

horns, BPH/5 embryos were crowded and clustered (Figure 1A). This was observed at a significantly higher rate than in C57 females during the window of implantation (E4.5-E5.5) (Supplemental Figure 1A; supplemental material available online with this article; doi:10.1172/jci.insight.75351DS1). Interestingly, embryo crowding is linked to unsynchronized embryo-uterine interactions, leading to abnormal placentation $(9,22$, 23). Notably, this spacing anomaly is not apparent in parental strains of $\mathrm{BPH} / 5$, such as $\mathrm{BPH} / 2$ and $\mathrm{BPN} / 3$ (ref. 24 and Supplemental Figure 1B). Moreover, embryo transfer (ET) studies were performed to determine the contribution of the embryo versus the uterine environment to the observed spacing anomaly. Only when $\mathrm{BPH} / 5$ embryos were transferred back into $\mathrm{BPH} / 5$ recipients did the embryos cluster abnormally along the uterine horns $(33.25 \pm 11.8 \%, n=4)$. As expected, C57 embryos in C57 recipients did not cluster $(0 \%, n$ $=3$ ). Interestingly, abnormal clustering was also not observed in reciprocal ET experiments: $\mathrm{C} 57$ embryos in $\mathrm{BPH} / 5$ recipients $(0 \%, n=4)$ and $\mathrm{BPH} / 5$ embryos in $\mathrm{C} 57$ recipients $(0 \%, n=3)$. This finding provided 
A
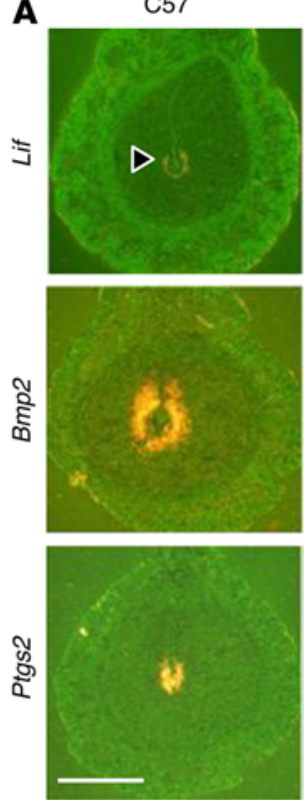

$\mathrm{BPH} / 5$
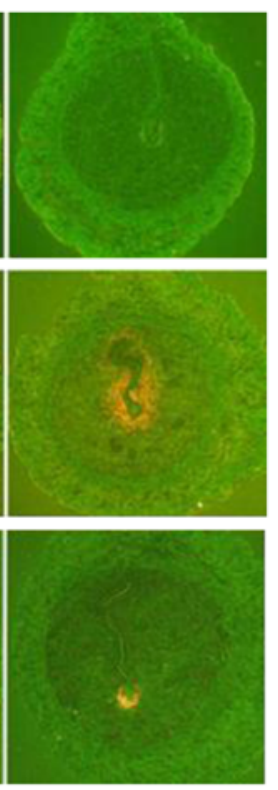
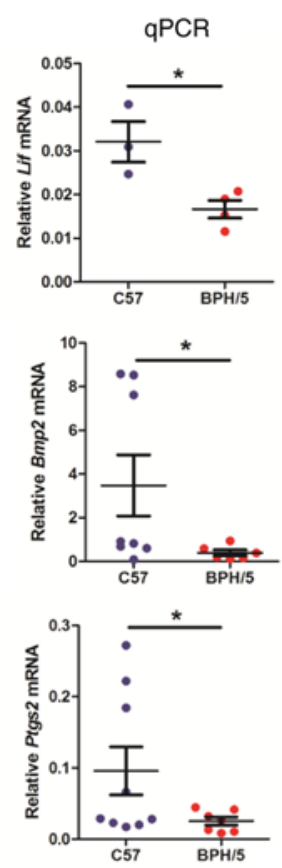

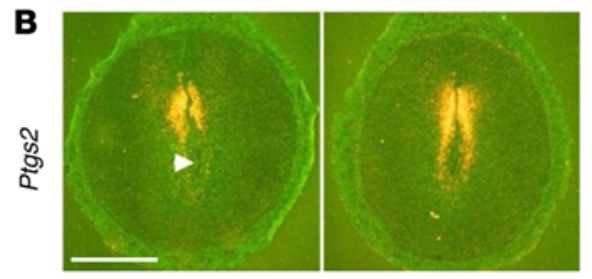

e5.5am of pregnancy

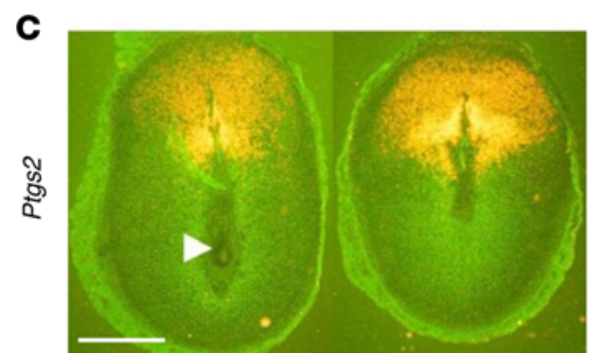

e7.5am of pregnancy

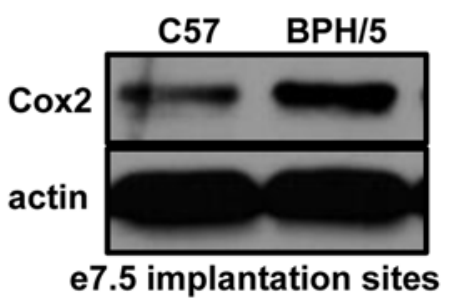

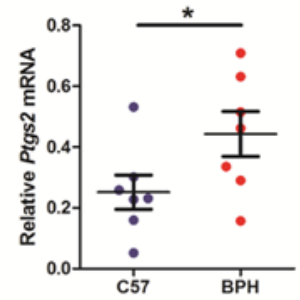

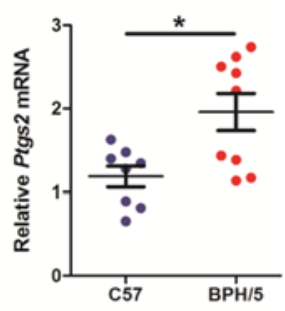

Western

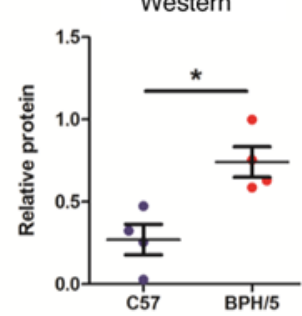

Figure 2. BPH/5 uteri show altered expression of molecules critical for implantation. (A and B) Representative ISH (left panels) and quantification by qRT-PCR (right panels) of key transcripts (Lif, Bmp2, and Ptgs2) in implantation sites at (A) E4.5am and (B) E5.5am. $n=3-9$. ${ }^{*} P<0.05$, by 1-tailed Student's $t$ test for qRT-PCR corresponding to ISH. (C) Representative ISH and quantification by qRT-PCR measurements of Ptgs2 in implantation sites at E7.5am. $n=7,{ }^{*} P<0.05$, by 1-tailed Student's $t$ test for qRT-PCR corresponding to ISH. Also shown are a representative Western blot and a quantitative summary of Cox2 protein expression (right lower panel) in implantation sites at E7.5. Data are expressed as Cox2 protein relative to actin. $n=4$. ${ }^{*} P<0.05$, 2-tailed Student's $t$ test. Data represent the mean \pm SEM. Black arrowhead in $\mathbf{A}$ indicates the location of the blastocyst; white arrowheads in $\mathbf{B}$ and $\mathbf{C}$ indicate the embryo. Scale bars: $500 \mu \mathrm{m}$. qRT-PCR, quantitative real-time PCR.

further evidence for $\mathrm{BPH} / 5$-specific embryo-uterine crosstalk impairment during early pregnancy.

These characteristics of the implantation phenotype in $\mathrm{BPH} / 5$ females led us to investigate whether embryo-uterine interactions occurred within the appropriate window of implantation (E3.5-E5.5). While blue bands were observed in C57 uteri in the evening of E3.5 with the initiation of implantation, blue bands were absent in BPH/5 mice at this time (Figure 1B). This was reflected in significantly fewer implantation sites in BPH/5 mice on the morning of E4.5 compared with that observed in C57 mice, but comparable numbers were seen in the 2 strains by E4.5pm (Figure 1B). These results demonstrate that implantation is deferred outside of the normal window in $\mathrm{BPH} / 5$ females.

Maternal factors contribute to peri-implantation abnormalities in BPH/5. We next further addressed the potential contribution of maternal factors to dysregulated peri-implantation events in $\mathrm{BPH} / 5 \mathrm{females}$. We examined preimplantation embryonic development by flushing embryos from the gravid uterus on the morning of E3.5. Approximately $85 \%$ of C57 embryos analyzed were in the blastocyst stage, while only approximately $15 \%$ of $\mathrm{BPH} / 5$ embryos had reached the blastocyst stage by this time. The majority of $\mathrm{BPH} / 5$ embryos were still at the morula stage (Figure $1 \mathrm{C}, \mathrm{C} 57 \times \mathrm{C} 57$; $\mathrm{BPH} / 5 \times \mathrm{BPH} / 5$ ). To determine whether the maternal uterine environment played a role in the delayed embryo development in $\mathrm{BPH} / 5$, reciprocal crosses between the 2 strains were performed ( $\mathrm{C} 57$ females $\times \mathrm{BPH} / 5$ males; $\mathrm{BPH} / 5$ females $\times$ C57 males), and the development of F1 hybrid embryos was analyzed as above. Hybrid embryos showed delayed maturation in BPH/5 mothers compared with those in C57 mothers (Figure 1C, BPH/5 × C57; $\mathrm{C} 57 \times \mathrm{BPH} / 5)$, providing evidence for maternal contributions in $\mathrm{BPH} / 5$ embryo development.

Upon implantation, uterine stromal cells undergo decidualization in response to the embryo $(5,7,25)$. By assessing alkaline phosphatase (ALP) activity, a well-known assay for measuring decidualization (26), 
A

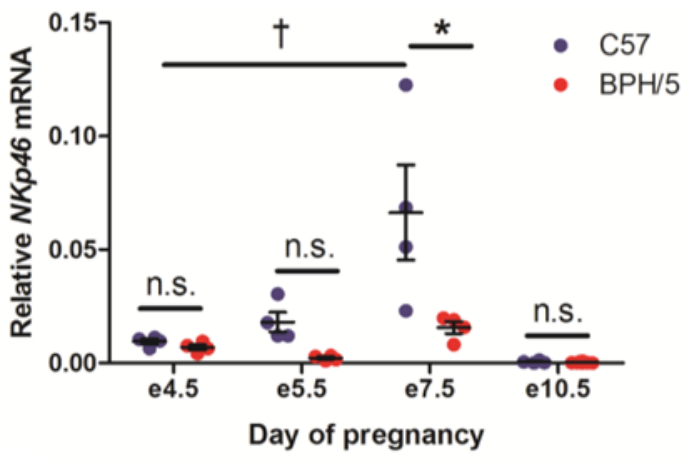

B

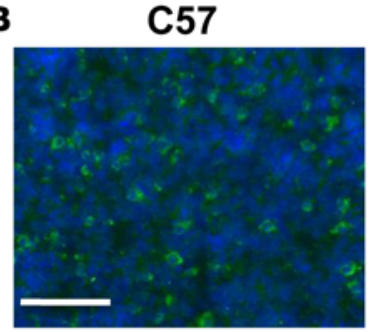

BPH/5

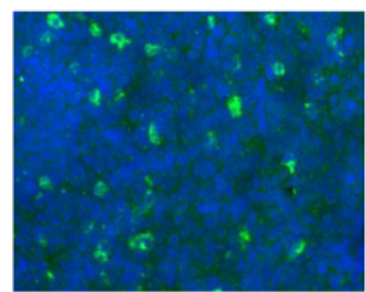

e7.5 of pregnancy

\section{C}

Flow Cytometry

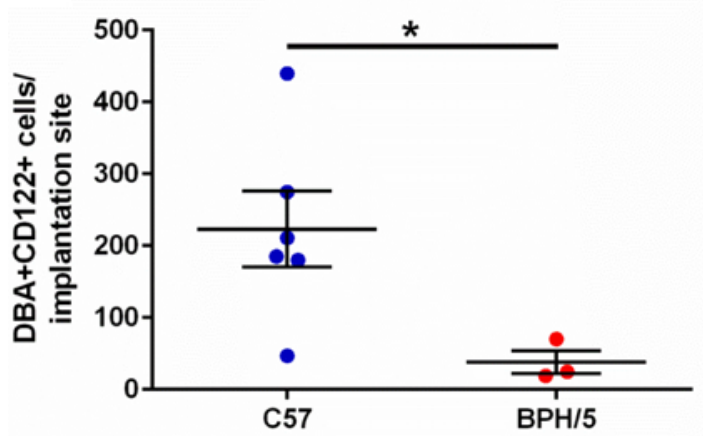

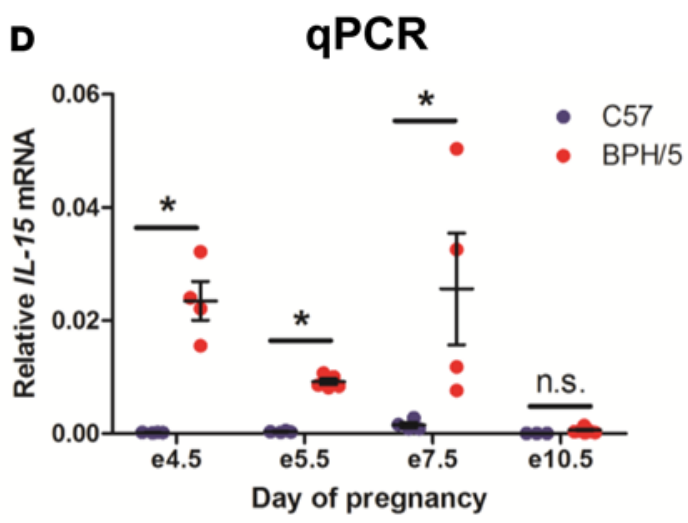

E

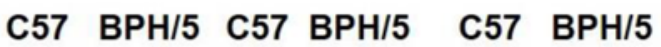

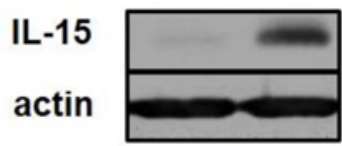

e4.5

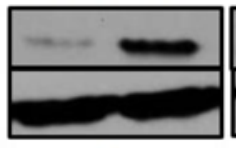

e5.5

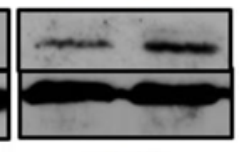

e7.5
$\mathbf{F}$

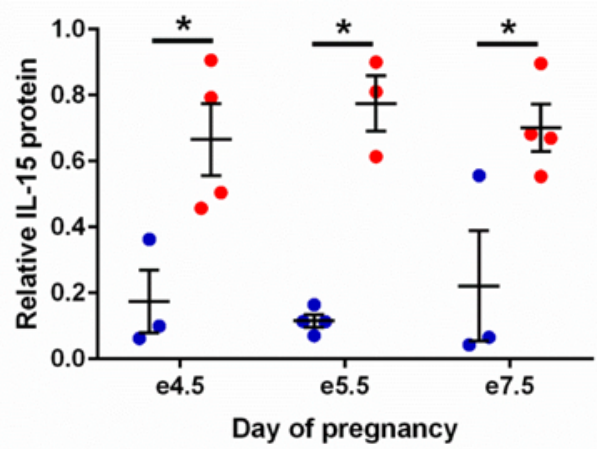

- $\mathrm{C} 57$

- $\mathrm{BPH} / 5$

Figure 3. Dramatic decreases in dNK cells are associated with increases in IL-15 at the maternal-fetal interface during the peri-implantation period in BPH/5 females. (A) Time course of qRT-PCR quantification of NKp46 mRNA in implantation sites collected at E4.5, E5.5, E7.5, and E10.5. $n=3-12$. * $P<0.05$ versus time-matched C57 mice and ${ }^{\dagger} P<0.05$ versus E4.5 C57 mice, by 2-way ANOVA. (B) Representative immunofluorescence of DBA ${ }^{+}$-stained cells in C57 and BPH/5 E7.5 implantation sites. Scale bar: $100 \mu \mathrm{m}$ (C) Quantitative summary of flow cytometry of DBA+CD122+-stained cells from E7.5 implantation sites. $n=3-6 .{ }^{*} P<0.05$, by 2-tailed Student's $t$ test. (D) Time course of qRT-PCR quantification of IL-15 mRNA in implantation sites collected at E4.5, E5.5, E7.5, and E10.5. $n=3-11 .{ }^{*} P<0.05$ versus time-matched C57 mice, by 2-tailed Student's $t$ test. (E) Representative Western blots of IL-15 protein expression as a time course in E4.5, E5.5, and E7.5 implantation sites. (F) Quantitative summary of IL-15 protein expression relative to actin. $n=3-4 .{ }^{*} P<0.05$ versus timematched C57 mice, by 2-tailed Student's $t$ test. Data represent the mean \pm SEM. dNK, decidual NK; qRT-PCR, qPCR, quantitative real-time PCR.

we observed dramatic differences in this process between BPH/5 and C57. While C57 showed robust ALP activity at E4.5am, this response was noticeably absent in BPH/5 implantation sites (Figure 1, D and E). This was followed by overcompensation of ALP activity at E4.5pm and E5.5am in BPH/5 compared with C57 mice (Figure 1, D and E). Thus, deferred implantation marked by embryo clustering in BPH/5 females was coincident with defective decidualization.

Since the circulating ovarian steroid hormones estrogen and progesterone $\left(\mathrm{P}_{4}\right)$ mediate uterine receptivity and therefore influence implantation (27), we measured levels of these pregnancy hormones in pregnant $\mathrm{BPH} / 5$ and $\mathrm{C} 57$ mice. Both the time course and levels of serum estradiol- $17 \beta\left(\mathrm{E}_{2}\right)$ during the preimplantation period (E2.5-E3.5) were significantly different between BPH/5 and C57 females (Supplemental Figure 2). At the time when $C 57$ mice showed the peak $E_{2}$ surge $(E 2.5 \mathrm{pm}), \mathrm{E}_{2}$ levels in $\mathrm{BPH} / 5$ mice were at their lowest and remained diminished at E3.5am compared with levels in C57 mice (Supplemental Figure 2A). 
Serum $\mathrm{P}_{4}$ levels were comparable between BPH/5 and C57 females, except at E2.5pm, when BPH/5 mice showed higher levels than did C57 mice (Supplemental Figure 2B). Supplementation with an exogenous $\mathrm{E}_{2}$ surge at $\mathrm{E} 2.5 \mathrm{pm}$ in $\mathrm{BPH} / 5$ females did not prevent the implantation delay (J.L. Sones, unpublished observations) at $\mathrm{E} 3.5 \mathrm{pm}$ (Figure $1 \mathrm{~B})$.

$\mathrm{BPH} / 5$ mice show dysregulated gene expression in the peri-implantation uterus. Coordinated embryo-uterine interactions leading to normal implantation require regulated signaling through multiple autocrine, paracrine, and juxtacrine factors $(5,7,25)$. To understand the molecular underpinnings of anomalous implantation events in $\mathrm{BPH} / 5$ females, we assessed the expression status of the following key signaling molecules in the uterus around the time of implantation and decidualization: homeobox A10 (Hoxa10), leukemia inhibitory factor (Lif), bone morphogenic protein 2 (Bmp2), and Cox2 (Ptgs2) (5, 28-30). Using both ISH and quantitative real-time PCR (qRT-PCR), our results show that signal intensities for Lif, Bmp2, and Ptgs2 were all lower in E4.5am BPH/5 implantation sites compared with C57 implantation sites (Figure 2A). Downregulation of Bmp2 and Ptgs2 in BPH/5 implantation sites at E4.5am provides further evidence of compromised decidualization, as marked by reduced ALP activity in BPH/5 females at this time point (see Figure 1, D and E). Interestingly, whereas Bmp2 and Hoxa10 were comparable in BPH/5 and C57 females by E5.5am (Supplemental Figure 3), Ptgs2 expression was aberrantly upregulated in BPH/5 females at this time point (Figure 2B). Overexpression of Ptgs2 in the developing decidua of BPH/5 mice persisted at E7.5am (Figure 2C), and these changes were reflected in Western blot analysis of Cox2 protein levels (Figure 2C). This is important, since Cox2 signaling may be a final common pathway upon which the implantation and decidualization upstream targets, including Lif, Hoxa10 and Bmp2, converge (7, 30). As such, we hypothesized that overexpression of uterine Cox 2 and higher prostanoid levels contribute to decidualization defects and propagation of adverse ripple effects affecting fetoplacental development in BPH/5 mice.

Dramatic decreases in $\mathrm{dNK}$ cells at the BPH/5 maternal-fetal interface due to IL-15 overexpression in the implantation site. Remodeling of decidual vessels is necessary for establishing uteroplacental perfusion, and dNK cells at the maternal-fetal interface aid in this process (11). As defects in spiral artery remodeling are a hallmark of $\mathrm{PE}$ in women (1), we next assessed this immune cell population during the peri-implantation period in BPH/5 mice. First, qRT-PCR was used to determine the mRNA expression pattern of NKp46, an activating receptor present in the gravid mouse uterus (31). During critical early pregnancy time points, $N K p 46$ mRNA increases with gestation in C57 implantation sites with peak expression at E7.5 (Figure 3A). $\mathrm{BPH} / 5$ implantation sites did not show this rise in $N K p 46$, but rather showed significantly lower expression at E7.5 versus C57 implantation sites (Figure 3A). We next measured dNK cell numbers by immunofluorescence and flow cytometry to see whether reduced NKp 46 mRNA levels within BPH/5 implantation sites at the time of maximal expression in C57 implantation sites (E7.5) corresponded to reduced staining of $\mathrm{DBA}^{+} \mathrm{CD} 122^{+}$cells, the dominant source of the Ifng transcript (32). As predicted, immunofluorescence localized $\mathrm{DBA}^{+}$cells to the decidua in both C57 and BPH/5 E7.5 implantation sites, with C57 implantation sites having more positive cells than BPH/5 implantation sites (Figure 3B). Flow cytometry confirmed that there were fewer $\mathrm{DBA}^{+} \mathrm{CD} 122^{+}$cells in $\mathrm{BPH} / 5$ implantation sites at E7.5 compared with the numbers in C57 implantation sites (Figure 3C and Supplemental Figure 4). Furthermore, $\mathrm{DBA}^{+} \mathrm{CD} 122^{+}$cells from these C57 implantation sites stained 3.5 times more intensely for IFN- $\gamma$ than did those from BPH/5 implantation sites (C57: $6 \% \pm 1.3 \%$ vs. BPH/5: $1.7 \% \pm 0.4 \%, n=4)$. Therefore, BPH/5 mice not only had fewer $\mathrm{dNK}$ cells at the maternal-fetal interface, but these dNK cells may also have had reduced function, as they appeared to produce less IFN- $\gamma$.

IL-15 is expressed by the pregnant uterus in mice and women (33) and is required for dNK cell activation (12). Since there is a dramatic reduction in $\mathrm{dNK}$ cells at the maternal-fetal interface in BPH/5 mice (Figure 3, A-C), we next assessed the expression of IL-15 in BPH/5 and C57 implantation sites. Using qRT-PCR, we saw significant upregulation of 1115 mRNA in BPH/5 implantation sites compared with C57 implantation sites beginning at E4.5, and this persisted at E5.5 and E7.5 (Figure 3D). Similarly, Western blot analyses showed that IL-15 protein was also significantly upregulated in BPH/ 5 implantation sites versus C57 sites at E4.5, E5.5, and E7.5 (Figure 3, E and F). This finding was unexpected, as IL-15 signaling promotes proliferation and maturation of dNK cells (33).

We next sought to functionally link overexpression of local IL-15 to dNK cell loss in C57 control mice. Pregnant C57 mice were anesthetized and given an intrauterine injection of a recombinant IL-15 Ab complex (IL-15R/IL-15Ra-Fc) or vehicle (saline) on E2.5 of pregnancy. Implantation sites were then collected at E5.5 to assess dNK cell activation status and dNK cell numbers within implantation sites. This treatment 
A

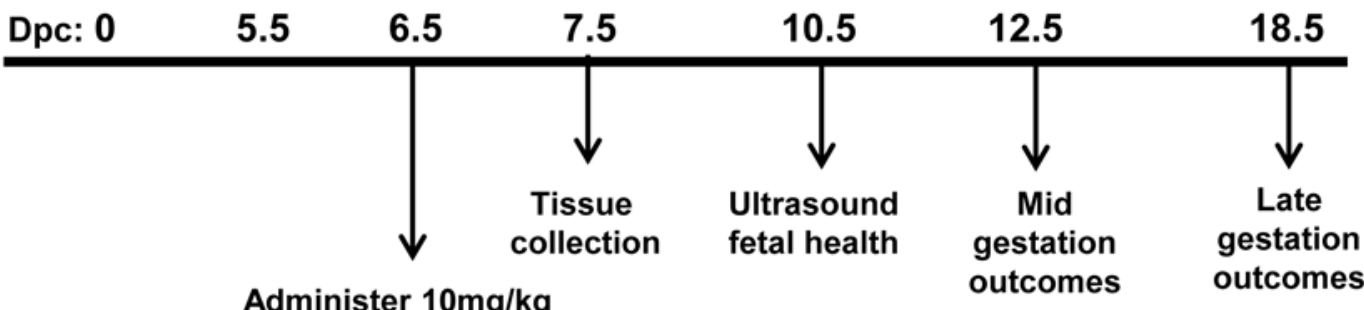

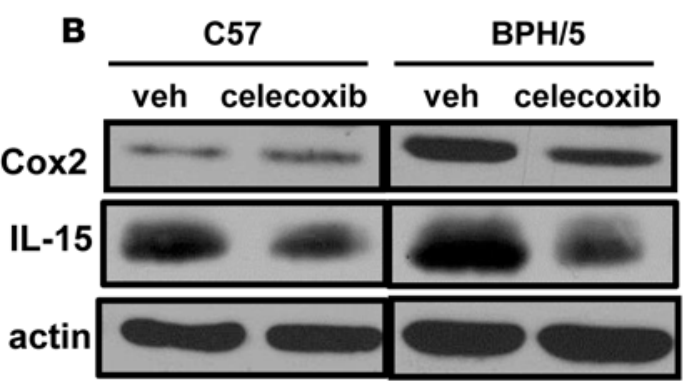

e7.5 implantation sites

\section{C}

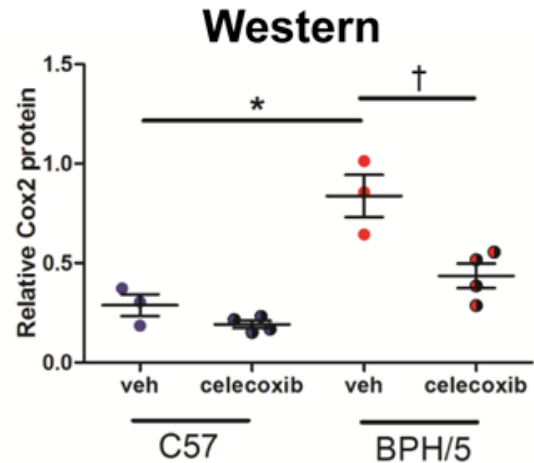

D

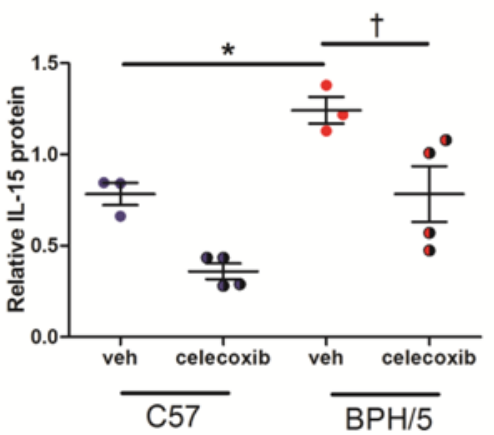

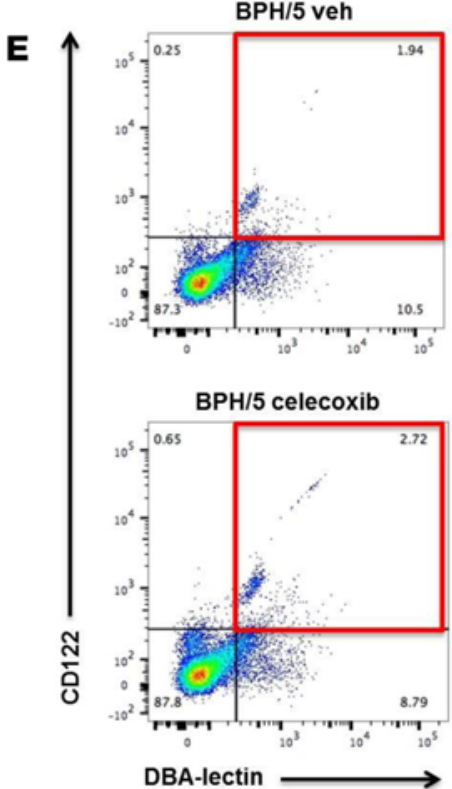

F $\quad$ Flow cytometry

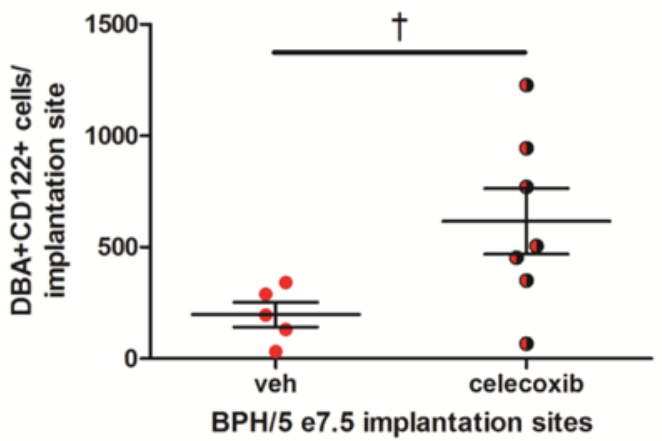

Figure 4. Celecoxib administration targets post-implantation Cox2 and IL-15 expression and restores dNK cell numbers in E7.5 BPH/5 implantation sites. (A) Schematic showing experimental strategy used to selectively inhibit Cox2. Celecoxib was administered by oral gavage on E6.5 after implantation but before peak decidualization (E7.5). (B) Representative Western blot of Cox2, IL-15, and actin in C57 E7.5 and BPH/5 E7.5 implantation sites. (C) Quantification of Cox2 protein and (D) IL-15 protein levels relative to actin in $\mathrm{C57}$ and BPH/5 E7.5 implantation sites after vehicle or celecoxib administration. $n=3$ for the vehicle-treated group; $n=4$ for the celecoxib-treated group. ${ }^{*} P<0.05$ versus vehicle-treated $\mathrm{C} 57$ mice and $\dagger P<0.05$ versus vehicle-treated $\mathrm{BPH} / 5$ mice, by 1-way ANOVA. (E) Representative dot plot of DBA+CD122+-stained cells from BPH/5 E7.5 implantation sites as measured by flow cytometry. Numbers in the boxes reflect the percentage of the total cells recovered that were found to be CD122+DBA-lectin ${ }^{+}$. (F) Quantitative summary of flow cytometry of DBA+CD122+-stained cells from BPH/5 E7.5 implantation sites for vehicle- $(n=5)$ versus celecoxibtreated mice. $n=7$. $† P<0.05$ versus vehicle-treated BPH/5 mice, by 2 -tailed Student's $t$ test. Data represent the mean \pm SEM. dNK, decidual NK; Dpc, days postcoitus; veh, vehicle. 
did not affect implantation, as we were able to visualize similar numbers of implantation sites between C57 vehicle and IL-15R/IL-15Ra-Fc-treated females (Supplemental Figure 5A). NKp46 mRNA levels were measured in implantation sites collected from both groups and found to be significantly decreased in the IL-15R/IL-15Ra-Fc-treated group (Supplemental Figure 5B). Furthermore, fewer DBA ${ }^{+} \mathrm{CD} 122^{+}$cells were observed by flow cytometry in C57 implantation sites from the IL-15R/IL-15Ra-Fc-treated group (Supplemental Figure 5C). Therefore, overexpression of IL-15 in the gravid uterus early in pregnancy plays a role in reducing $\mathrm{dNK}$ cell numbers at the maternal-fetal interface. This finding is supported by previously published work showing that sustained IL-15R/IL-15Ra-Fc in vivo resulted in impaired activation of mature NK cells in circulation (34).

Selective pharmacologic inhibition of Cox2 with celecoxib normalizes $I L-15$ and restores $d N K$ cells at the maternal-fetal interface early in BPH/5 pregnancy. Since appropriate Cox2 expression is pivotal for decidualization, placentation, and placental angiogenesis $(5,7,25,28)$, we tested the effect of early administration of the selective Cox2 inhibitor celecoxib in pregnant $\mathrm{BPH} / 5$ mice. We chose to administer celecoxib on E6.5 so as to not interfere with implantation and early decidualization, since Cox 2 is essential for these processes, a timing that allowed us instead to target misregulated Cox 2 during peak decidualization (ref. 35 and Figure $4 \mathrm{~A})$. First, we confirmed that our dose of celecoxib did not negatively impact implantation status in C57 or BPH/5 females (Supplemental Figure 6A). As observed by others (36), Cox2 protein levels were normalized after celecoxib administration in BPH/5 E7.5 implantation sites 24 hours after treatment (Figure 4, B and $\mathrm{C}$ ). We also tested the downstream effects of Cox2 inhibition by measuring prostaglandin $\mathrm{E}_{2}\left(\mathrm{PGE}_{2}\right)$ and 6-keto $\mathrm{PGF}_{1 \alpha}$ levels in $\mathrm{BPH} / 5$ and $\mathrm{C} 57$ implantation sites at E7.5 after administration of celecoxib or vehicle. Celecoxib reduced $\mathrm{PGE}_{2}$ levels in $\mathrm{BPH} / 5$ implantation sites compared with levels in vehicle-treated sites (Supplemental Figure 6B), with a trend toward reduced 6-keto PGF P lo $_{1}$ levels (Supplemental Figure 6C). These results indicated that celecoxib effectively reduced aberrant Cox2 expression at E7.5. Interestingly, celecoxib administration also normalized IL-15 protein levels in BPH/5 E7.5 implantation sites to the levels detected in C57 implantation sites from vehicle-treated mothers (Figure 4, B and D). This unexpected finding was associated with an increase in $\mathrm{DBA}^{+} \mathrm{CD} 122^{+} \mathrm{dNK}$ cells in $\mathrm{BPH} / 5$ E7.5 implantation sites from celecoxib-treated versus vehicle-treated mothers (Figure 4, E and F). These data demonstrated that celecoxib administration at E6.5 not only normalized Cox2 and $\mathrm{PGE}_{2}$ levels in the $\mathrm{BPH} / 5$ implantation sites, but also reduced IL-15 overexpression and restored dNK cell numbers at the maternal-fetal interface a mere 24 hours after treatment.

Celecoxib early in pregnancy improves adverse pregnancy outcomes in BPH/5 mice. We have previously shown by ultrasonography that $\mathrm{BPH} / 5$ fetuses exhibit varying statuses at mid gestation, ranging from healthy to compromised to resorbed (15). This is associated with smaller litter sizes and low-birth-weight pups $(15,16)$. Using a similar approach, we confirmed this variation in fetal health as early as E10.5 in BPH/5 females (Supplemental Table 2 and Figure 5A, vehicle). To test the hypothesis that dysregulated Cox 2 and IL-15 during the peri-implantation period in $\mathrm{BPH} / 5$ mice has a significant effect on downstream fetoplacental health, celecoxib was given to pregnant females by oral gavage on E6.5. Ultrasound studies were performed at E10.5, and females were assessed for litter size and placental and fetal weights at E12.5 and E18.5 (Figure 4A). BPH/5 females treated with celecoxib showed significant improvement in overall litter health at E10.5, with a significant increase in the percentage of healthy fetuses and a marked reduction in the percentage of compromised fetuses (Figure 5B). At this time point, the percentage of resorbed fetoplacental units per litter was unchanged from that of vehicle-treated $\mathrm{BPH} / 5$ females (Figure 5B). Intriguingly, we observed a significant reduction in the percentage of resorptions per BPH/5 litter at E12.5, with increased BPH/5 litter size (Figure 5, C and D). Furthermore, celecoxib normalized placental and fetal weights in BPH/5 females at E12.5 (Figure 5, E and F).

Improved fetoplacental development was also observed at late gestation in BPH/5 females. We have previously described profound fetoplacental defects in BPH/5 mice, including in utero FGR and inadequate decidual vessel remodeling (16). These 2 key preeclamptic features were reversed in $\mathrm{BPH} / 5$ mice with improvement in pup weight (Figure 6, A and B) and increased luminal diameters of decidual vessels at E18.5 after celecoxib treatment as compared with vehicle-treated mice (Figure 6, C and D). Most intriguing, celecoxib given early in pregnancy to $\mathrm{BPH} / 5$ mothers significantly reduced the late gestation (E15.5$\mathrm{E} 18.5$ ) rise in mean arterial pressure (MAP) observed in this model (Figure 6E). This finding is highly significant, as it identifies a mechanism by which early antiinflammatory treatment reduces the incidence of PE and FGR in high-risk pregnancies (19). Together, these data support our hypothesis that the FGR 
A

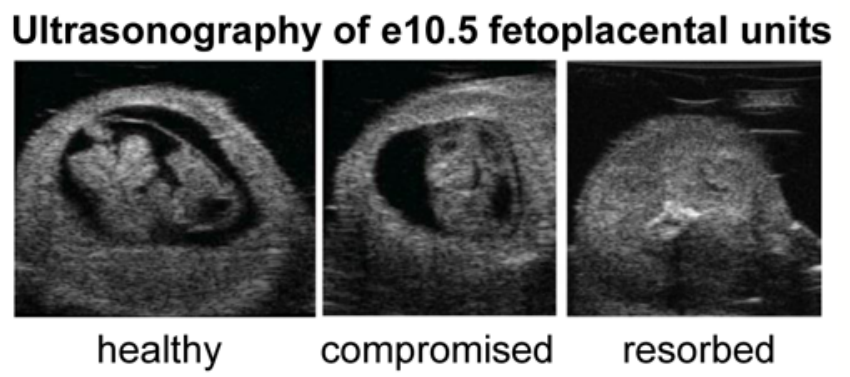

B

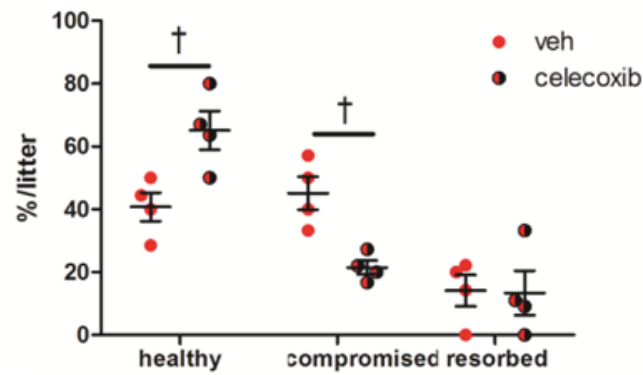

D
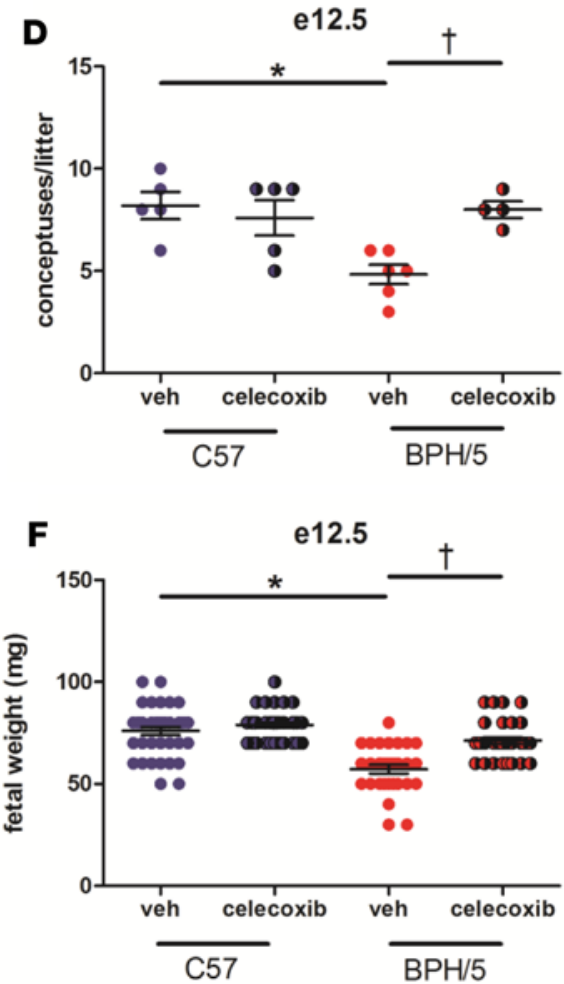

Figure 5. Selective inhibition of Cox2 early in pregnancy improves mid-gestation fetoplacental outcomes in BPH/5 females. (A) Ultrasonography was used to identify 3 distinct morphologic classes of fetal health in BPH/5 females at E10.5: healthy, compromised, and resorbed. (B) Assessment of fetal health in BPH/5 females at E10.5 via ultrasound after vehicle or celecoxib administration. $n=4$ litters/group. ${ }^{\dagger} P<0.05$ versus vehicle-treated BPH/5 mice, by 2-way ANOVA. (C) Resorptions were observed and recorded at E12.5 as a percentage of resorptions per litter. $n=4-6$ litters/group. ${ }^{*} P<0.05$ versus $C 57$ vehicle-treated $\mathrm{C} 57$ mice and ${ }^{\dagger} P<0.05$ versus vehicle-treated $\mathrm{BPH} / 5$ mice, by 1-way ANOVA. (D) Litter size was counted in BPH/5 and C57 mice at E12.5 after administration of vehicle ( $n=6 \mathrm{BPH} / 5$ females and $n=5 \mathrm{C} 57$ females) or celecoxib ( $n=4 \mathrm{BPH} / 5$ females and $n=5 \mathrm{C} 57$ females). ${ }^{*} P<0.05$ versus vehicle-treated $\mathrm{C} 57$ mice and ${ }^{\dagger} P<0.05$ versus vehicle-treated $\mathrm{BPH} / 5$ mice, by 1-way ANOVA. (E) Placental weight and (F) fetal weight were measured in BPH/5 and C57 mouse pregnancies at E12.5 after administration of vehicle ( $n=29 \mathrm{BPH} / 5$ mice and $n=40$ C57 mice) or celecoxib ( $n=32 \mathrm{BPH} / 5 \mathrm{mice}$ and $n=36$ C57 mice). ${ }^{*} P<0.05$ versus vehicle-treated $\left[57\right.$ mice and ${ }^{\dagger} P<0.05$ versus vehicle-treated $\mathrm{BPH} / 5$ mice, by 1 -way ANOVA. Data represent the mean $\pm \mathrm{SEM}$. veh, vehicle.

and maternal hypertension associated with $\mathrm{PE}$ in $\mathrm{BPH} / 5$ females begin early in pregnancy and emphasize the need to identify high-risk pregnancies as early as possible.

\section{Discussion}

This study clearly demonstrates that dysregulation of early pregnancy events (implantation and decidualization) underlie the deleterious fetal and maternal pregnancy outcomes observed in the BPH/5 mouse model of spontaneous PE (Figure 7). Although abnormal implantation and placentation have been linked to the pathogenesis of PE, the definitive mechanisms are still unknown $(1,20,37)$. Early events associated with PE have been difficult to study in humans due to logistical and ethical challenges. Although rodent models are widely used to study the pathophysiology of PE, many rely on mechanical or pharmacological 
A

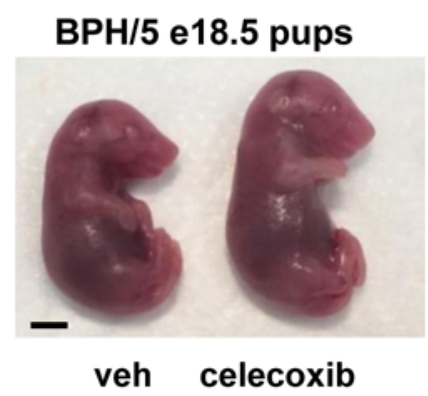

C

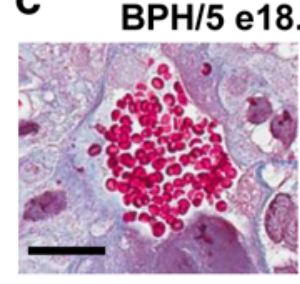

veh

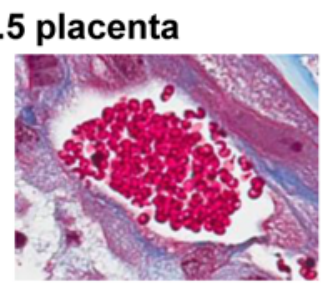

celecoxib

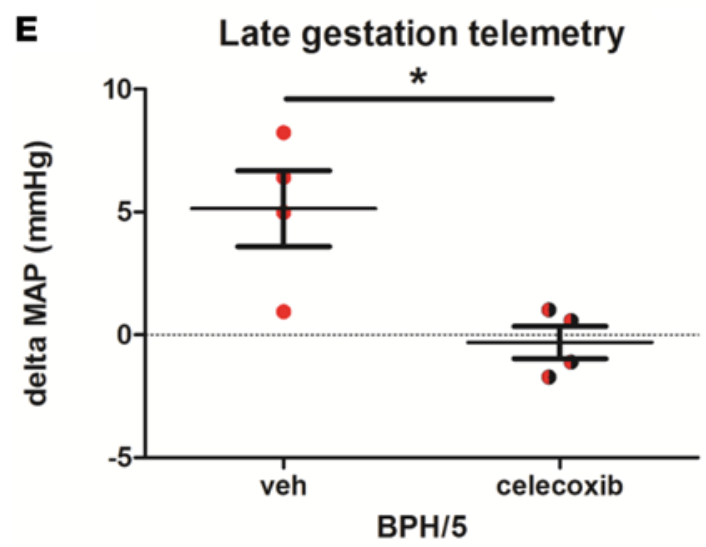

B

Pup weight

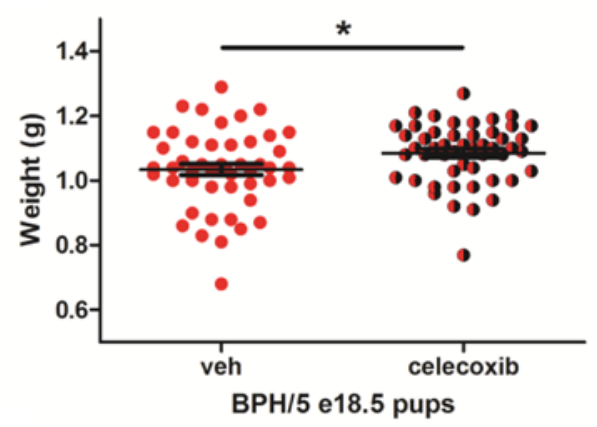

D Decidual vessels

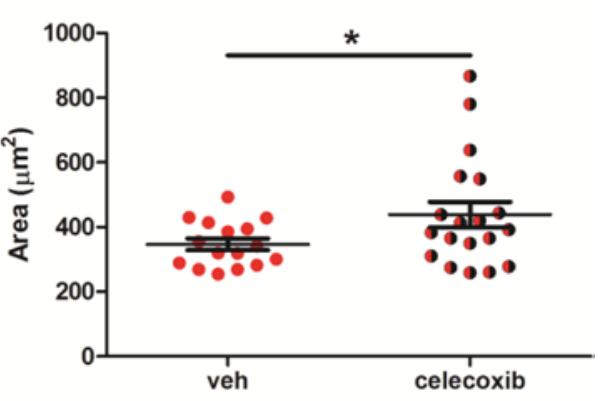

BPH/5 e18.5 placenta

Figure 6. Late-gestational BPH/5 fetoplacental outcomes have sustained improvement, and the maternal PE syndrome is partially ameliorated after early Cox2 inhibition with celecoxib. (A) Representative image of BPH/5 pup size at E18.5 after vehicle or celecoxib administration. Scale bar: $4 \mathrm{~mm}$. (B) BPH/5 pup weight was measured at E18.5 after administration of vehicle $(n=49)$ or celecoxib $(n=51) .{ }^{*} P<0.05$ versus vehicle-treated BPH/5 mice, by 2 -tailed Student's $t$ test. (C) Representative images of Masson's trichrome-stained BPH/5 placenta at E18.5 after vehicle or celecoxib administration. Scale bar: $25 \mu \mathrm{m}$. (D) Area of decidual vessels was calculated for BPH/5 placenta taken at E18.5 after vehicle $(n=16)$ or celecoxib $(n=19)$ administration. ${ }^{*} P \leq 0.05$ versus vehicle-treated $\mathrm{BPH} / 5$ mice, by 2 -tailed Student's $t$ test. (E) $\triangle \mathrm{MAP}$ was measured using radiotelemetry and reported relative to baseline nonpregnant MAP during late gestation (E15.5-E18.5) in BPH/5 mothers treated with vehicle $(n=6)$ or celecoxib $(n=6) .{ }^{*} P=0.017$, by 2-tailed Student's $t$ test. Data represent the mean \pm SEM. MAP, mean arterial pressure; veh, vehicle.

manipulations at mid gestation to induce the late gestational maternal syndrome $(38,39)$. Therefore, animal models that spontaneously mimic PE in women are vital to understanding early events in pregnancy that lead to the eventual presentation of the maternal syndrome. In this study, we investigated peri-implantation defects in $\mathrm{BPH} / 5$ mice and present evidence to support our hypothesis that aberrations in early pregnancy events influence the fetoplacental and maternal outcomes associated with the PE syndrome. Furthermore, our data implicate overexpressed uterine Cox2 and IL-15 as causal factors in the poor fetoplacental development and maternal health status in $\mathrm{BPH} / 5$ pregnancies.

Implantation in BPH/5 females occurs beyond the normal window of implantation, with abnormal spacing of implantation sites (embryo clustering) and defective decidualization compared with that seen in C57 females. Wilcox et al. observed an increased incidence of early pregnancy loss in humans when 


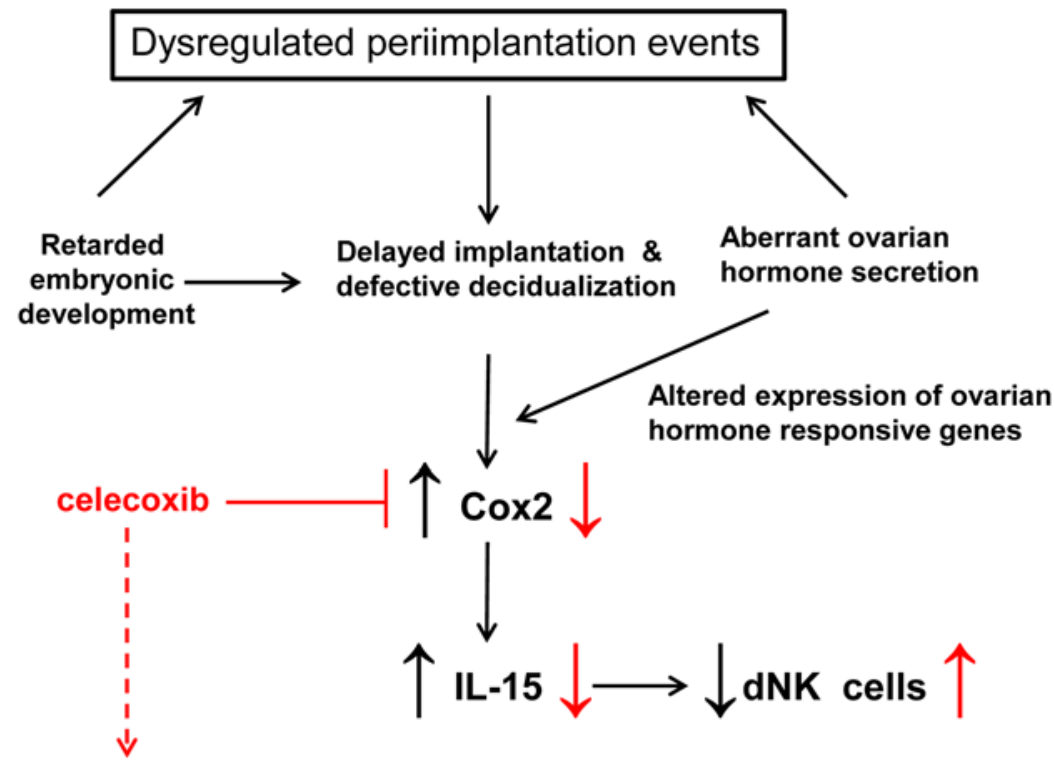

Improved FGR, placental vascular

development, \& maternal hypertension

Figure 7. Proposed scheme for Cox3 regulation of fetoplacental outcomes at the maternal-fetal interface in BPH/5 females. Dysregulated peri-implantation events in $\mathrm{BPH} / 5$ culminated in the robust upregulation of Cox2 at the maternal-fetal interface of $\mathrm{BPH} / 5$ females early in pregnancy (E7.5). This was associated with a dramatic reduction in $\mathrm{dNK}$ cell numbers and overexpression of IL-15 protein in E7.5 BPH/5 implantation sites as compared with $\mathrm{C57}$ implantation sites. Normalization of Cox2 and IL-15 protein levels with the selective Cox2 inhibitor celecoxib improved dNK cell numbers at the maternal-fetal interface in BPH/5 mice at E7.5 as well as fetal, placental, and maternal outcomes. dNK, decidual NK; FGR, fetal growth restriction.

implantation occurred outside the prescribed window of uterine receptivity (40); however, the association of deferred implantation with adverse pregnancy outcomes such as PE has not been defined in humans. Interestingly, we also observed retarded development of $\mathrm{BPH} / 5$ embryos as well as of $\mathrm{BPH} / 5 \times \mathrm{C} 57$ hybrid embryos in BPH/ 5 mothers, but not in $\mathrm{C} 57$ mothers. The disparate $\mathrm{E}_{2}$ levels between $\mathrm{C} 57$ and $\mathrm{BPH} / 5$ mice prior to implantation led us to speculate that the expression of genes involved in uterine receptivity may also be dysregulated in BPH/5 females. Expression of Lif and Msx1, markers of uterine receptivity (41-43), were evaluated by ISH and qRT-PCR in E3.5am uteri. Despite the abnormal $\mathrm{E}_{2}$ levels in BPH/5 females, Lif and Msx 1 had comparable expression patterns in BPH/5 and C57 uteri (J.L. Sones, unpublished observations). However, other significant factors required for uterine receptivity may display differential expression (44) and have yet to be explored in $\mathrm{BPH} / 5$ mice.

Interestingly, lower circulating levels of $\mathrm{E}_{2}$ in $\mathrm{BPH} / 5$ females were associated with reduced expression of Lif, an $\mathrm{E}_{2}$-responsive gene, at E4.5am. Reduced induction of Bmp2 and Ptgs2 during early decidualization, but overexpression of Ptgs2 during maximal decidualization (E5.5-E7.5), in BPH/5 females clearly show that the decidualization process was aberrant.

Compensation of Ptgs2 expression in BPH/5 implantation sites at E7.5 alludes to the critical nature of appropriate Cox 2 signaling in pregnancy outcome. Indeed, Ptgs $2^{-1-}$ females showed failure in several pregnancy events including ovulation, fertilization, implantation, and decidualization (28). Furthermore, Cox2-derived prostanoids are necessary for uterine angiogenesis during decidualization and are also responsible for regulating fetal and placental vascularity $(8,45)$. Interestingly, we have shown that $\mathrm{BPH} / 5$ mice exhibit dysregulated placental VEGF levels and that VEGF gene therapy at E7.5 ameliorated some aspects of the PE syndrome in this model (18). We speculate that dysregulated Cox 2 expression may be contributing to these detrimental vascular events during placental development in BPH/5 females, and these studies require further investigation. Preliminary data suggest that celecoxib administration restores the angiogenic imbalance with regard to VEGF protein expression at the maternal-fetal interface. These data, along with the data presented herein, support the concept that balanced expression of molecular mediators of implan- 
tation and decidualization (Cox2 and Cox2-derived prostanoids) are central to placentation, fetal growth and development, and overall pregnancy success.

Investigation of the role of $\mathrm{dNK}$ cells in $\mathrm{PE}$ has gained importance in recent years. Not only are $\mathrm{dNK}$ cells the most abundant immune cell at the maternal-fetal interface, constituting approximately $70 \%$ of the immune cell population, they also have important regulatory functions during pregnancy (11). They are highly involved in 2 developmental processes crucial for placentation, trophoblast invasion, and vascular remodeling (46). Term placentae from both PE pregnancies and pregnancies with FGR alone have been reported to contain fewer $\mathrm{dNK}$ cells in the decidua (47). However, dNK cells reach maximal expression in the human placenta by the end of the second trimester (33). Thus, analyses of dNK cell populations taken from placentae at the time of delivery do not adequately interrogate their contributions in PE. Using NKp46 as a marker of activated dNK cells, we discovered that C57 mice show a progressive increase in implantation site $N K p 46$ expression that peaks at E7.5. BPH/5 mice did not show this rise in expression and had significantly lower $N K p 46$ expression levels compared with C57 mice at this critical time point in pregnancy, suggesting that the previously described defects in $\mathrm{BPH} / 5$ decidual vessel remodeling (16) may be due to dNK cell deficiency.

Uterine IL-15 is physiologically upregulated at the time of decidualization to activate dNK cells (33) As with BPH/5 mice, $I l 15^{-1-}$ mice show inappropriate decidualization, lack mature $\mathrm{dNK}$ cells, and, interestingly, produce low-birth-weight pups (48). Exogenous IL-15 administration to these mice restores dNK cell populations, thus confirming its importance in $\mathrm{dNK}$ cell maturation. We unexpectedly found that $\mathrm{BPH} / 5$ implantation sites show robust expression of both IL-15 mRNA and protein at E4.5, E5.5, and E7.5 compared with C57 implantation sites. This robust overexpression may be a consequence of defective decidualization or a compensatory response of the uterus to produce more IL-15 to recruit adequate pre-dNK cells to the BPH/5 decidua. Increased decidual IL-15 expression has been linked to recurrent miscarriage in women, suggesting impaired implantation and vascularization of the placenta (49). Other reports have demonstrated increased circulating IL-15 levels in the serum of preeclamptic mothers as compared with levels in healthy controls, in whom IL-15 levels were proportional to the severity of disease presentation (50). These findings provide evidence that IL-15 may be involved in poor pregnancy outcomes. Because $\mathrm{BPH} / 5$ females had consistently higher levels of IL-15 in the implantation site as compared with C57 females during peri-implantation time points (Figure 3, D and F), we were curious to determine IL-15 levels in the BPH/ 5 uterus before pregnancy. Indeed, Il15 mRNA levels were upregulated by nearly 85 -fold in the BPH/5 nongravid uterus compared with levels in C57 females (BPH/5: $2.53 \pm 0.76, n=8$ versus C57:0.03 $\pm 0.01, n=6$ ). Therefore, further work is needed to determine the precise mechanism of IL-15 overexpression in $\mathrm{BPH} / 5$ females as well as the targeting strategies to normalize its expression.

To test experimentally whether IL-15 overexpression is linked to dNK cell loss, C57 mice were given an intrauterine injection of a recombinant IL-15-Ab complex (IL-15R/IL-15Ra-Fc) on E2.5 to increase local IL-15 expression at the time of implantation. Strikingly, NKp46 mRNA was reduced and DBA $\mathrm{CD} 122^{+}$cells were less abundant compared with levels detected in C57 vehicle-treated mice, suggesting a direct effect of local IL-15 on dNK cell activation. IL-15 is crucial for dNK cell-mediated adaptations in establishing placental blood flow (33). Some reports describe increased placental dNK cells at delivery in patients with PE who also have increased serum levels of IL-15 (51). Furthermore, these dNK cells have an altered phenotype that may be responsible for the dysregulated dNK signaling in these tissues. This would suggest that the $\mathrm{dNK}$ phenotype would be important to examine in $\mathrm{BPH} / 5$ mice and could provide even further insight into the altered immunoregulatory mechanisms during PE pregnancies. Assessing the angiogenic potential of $\mathrm{BPH} / 5 \mathrm{dNK}$ cells and whether they produce adequate VEGF could provide further mechanistic insight into the early angiogenic imbalance seen in $\mathrm{BPH} / 5$ mice. Also, ovarian hormones are thought to play a prominent role in this recruitment process (33). $\mathrm{BPH} / 5$ females have aberrations in ovarian hormone profiles during pregnancy as well as in the nonpregnant state (J.L. Sones, unpublished observations). Further investigation of $\mathrm{dNK}$ cell recruitment to the decidua and the endocrine profile in $\mathrm{BPH} / 5$ mice is thus warranted.

To causally link our findings that peri-implantation defects and Cox2 signaling play a key role in initiating downstream fetoplacental defects in this mouse model of spontaneous PE, we devised a strategy to target the dysregulated Cox 2 at the peak of decidualization. Selective inhibition of Cox 2 by celecoxib given to pregnant mice at E6.5 markedly improved fetal and maternal pregnancy outcomes at mid and late gestation in $\mathrm{BPH} / 5$ mice, with a significant reduction in Cox 2 protein and $\mathrm{PGE}_{2}$ levels in $\mathrm{BPH} / 5$ implanta- 
tion sites. This treatment did not impair implantation, indicating appropriate dose and timing of celecoxib administration for improvement in pregnancy outcomes. These results are intriguing, since altered levels of Cox2-derived prostanoids are implicated in the pathogenesis of PE and in the abnormal umbilical blood flow seen in women with pregnancy-induced hypertension and PE $(52,53)$. Interestingly, our treatment strategy not only reduced $\mathrm{PGE}_{2}$ levels, but also Cox2 protein levels. Experiments using stable cell lines have shown that celecoxib-mediated downregulation of $\mathrm{NF}-\kappa \mathrm{B}$ results in decreased Cox 2 promoter activity (36).

One study using fibroblast-like synoviocytes taken from patients with rheumatoid arthritis showed that Cox2 is increased by IL-15 in a $\mathrm{PGE}_{2}$-dependent manner (54). Consistent with this study, we show that Cox2 inhibition with celecoxib reduced IL-15 protein levels in BPH/5 celecoxib-treated compared with levels in vehicle-treated implantation sites (Figure 4, B and D). These data suggest that $\mathrm{PGE}_{2}$ may have feedback on IL-15 to lower IL-15 expression levels and the subsequent expression levels of Cox 2 following celecoxib administration, contributing to improved pregnancy outcomes. NF- $\kappa \mathrm{B}$ is one potential upstream regulator of both IL-15 and Cox2 (54). Inhibition of Cox2, prostaglandins, and NF- $\mathrm{B}$ has already been proposed as a potential therapeutic approach to treat preterm labor (55). Further studies are required to evaluate the regulatory mechanisms that involve IL-15 and Cox2.

Finally, improved fetal and placental outcomes in BPH/5 pregnant mice with respect to increased pup weight and decidual vessel size, respectively, following correction of aberrant Cox 2 levels by oral celecoxib administration provides evidence for a causal role of abnormal decidual Cox 2 signaling in the FGR and placentopathies associated with PE. Additionally, amelioration of maternal hypertension late in gestation further underscores the significance of early events in PE pregnancy outcomes. Although peri-implantation anomalies show adverse ripple effects on fetoplacental development and pregnancy outcomes (5), it has been difficult to study the impact of these early events in the context of PE. Here, we show that dysregulation of embryo-uterine events during the peri-implantation period results in undesirable fetal and pregnancy outcomes in a mouse model of PE. These effects are mediated by Cox 2 and IL-15 in abnormal fetal growth, placental development, and maternal hypertension in pregnancies affected by PE, making celecoxib a potential therapeutic for preventing the fetal morbidity and mortality and maternal hypertension that are associated with PE and perhaps other disorders of pregnancy (56).

\section{Methods}

For additional details on methods, see the Supplemental Methods.

Mice. Virgin BPH/5 and control C57B1/6 (C57) mice (8-12 weeks of age) were obtained from in-house colonies. $\mathrm{C} 57$ mice have been used as control mice in previous BPH/5 studies, as they were used in the original 8-way cross to derive the $\mathrm{BPH} / 5$ strain (24). Strain-matched and inter-strain matings (reciprocal crosses) were performed with BPH/5 and C57 males (8-12 weeks of age) to induce pregnancy. The day of vaginal plug detection was defined as E0.5. Celecoxib (Sigma-Aldrich) was administered to pregnant mice at E6.5, as previously described (57).

Analysis of pregnancy outcomes. Preimplantation embryo development, implantation, and mid- and late-gestational outcomes were assessed according to previously published protocols $(17,41,58)$.

ALP activity assay. To assess the decidualization of uterine tissues, ALP activity assays were performed on implantation sites as previously described (26).

Hormone assays. Blood was collected via cardiac puncture, allowed to clot at room temperature for 90 minutes, and centrifuged at 3,500 rpm for 20 minutes. Serum was frozen at $-80^{\circ} \mathrm{C}$ until assayed. A commercially available estradiol ELISA was performed according to the manufacturer's instructions (Calbiotech). The sensitivity of this assay is $3 \mathrm{pg} / \mathrm{ml}$.

Ultrasound assessment of fetoplacental health. Vevo 770 ultra-high-frequency ultrasound (Visualsonics) was used to examine fetal health in pregnancy as previously described $(15,59)$.

$R N A$ isolation and quantitative RT-PCR. Total RNA was extracted, and qRT-PCR was performed as previously described (60) using the primer sequences listed in Supplemental Table 1.

ISH. ISH was performed on implantation sites as previously described (28).

Western blotting. Protein was measured in implantation sites according to previously published methods (18). Band intensity was measured by densitometric analysis and expressed relative to actin.

Measurement of prostaglandins. The amount of $\mathrm{PGE}_{2}$ and prostacyclin $\left(\mathrm{PGI}_{2}\right.$; stable metabolite 6-keto prostaglandin $\mathrm{F}_{1 \alpha}$ ) in each sample was measured by ELISA according to the manufacturer's instructions (Cayman Chemicals) and as previously described (61). 
Immunofluorescence microscopy. Sections $(10-\mu \mathrm{m})$ were taken through C57 and BPH/5 implantation sites. Sections with the embryo were identified, blocked using serum-free blocking buffer for 1 hour, and stained with DBA-lectin (1:500) overnight at $4^{\circ} \mathrm{C}$ in serum-free blocking buffer, followed by DAPI staining $(1: 5,000)$ for 5 minutes, and were then mounted using prolong gold mounting media. Slides were imaged at $\times 20$ magnification, with exposure times of $872 \mathrm{~ms}$ for DBA-lectin and $14 \mathrm{~ms}$ for DAPI.

Flow cytometry. Single-cell suspensions were made from dissected implantation sites at E7.5 and counted using trypan blue exclusion. Cells were stained for viability using Fixable Viability Stain 510 (BD Horizon). Cells were subsequently washed in FACS buffer (1× PBS, 1\% BSA, 1 mM EDTA) and blocked with Fc block (purified anti-CD16/CD32 [2.4G2]) (BD Pharmingen) for 10 minutes at $4^{\circ} \mathrm{C}$. Surface markers were stained using the following fluorophore-conjugated Abs, as previously described (31): Per-CP-CD45 (30-F11), PE-CD122 (TM- $\beta 1$ ), and APC-TCR- $\beta$ (H57-597) (all from BD Pharmingen). FITC-conjugated Dolichos biflorus agglutinin (DBA) lectin (US Biological) was also used as a cell-surface marker. Flow cytometric analysis was performed using an LSR II Flow Cytometer (BD) and FlowJo software (Tree Star). Gates were determined by comparing each sample to a no-DBA-lectin, PE-IgG2b control. Count Bright counting beads (Molecular Probes) were used to determine the number of CD $122^{+}$DBA-lectin ${ }^{+}$cells per implantation site.

Intrauterine delivery of recombinant IL-15. A recombinant IL-15-Ab complex (IL-15R/IL-15Ra-Fc; R\&D Systems) was administered to C57 female mice at E2.5 by intrauterine injection as previously described (62). Two days after positive plug detection, C57 females were anesthetized with an i.p. injection of ketamine $(100 \mathrm{mg} / \mathrm{kg})$ and xylazine $(10 \mathrm{mg} / \mathrm{kg})$. Two dorsal incisions were made over the ovarian fat pads. The uterine horns were identified and elevated by manipulating the ovarian fat pad. IL-15R/IL-15Ra-Fc (15 $\mu \mathrm{g})$ was dissolved in $20 \mu \mathrm{l}$ saline, divided into 2 aliquots, and injected into the distal tip of each uterine horn. Control mice were given similar intrauterine injections of saline. For the analysis of outcomes, C57 females were allowed to recover and were sacrificed 3 days later at E5.5 to collect implantation sites.

Histological analyses. Conceptuses were harvested at E18.5 from BPH/5 mice after vehicle or celecoxib treatment. The placenta was dissected free from the fetus and fixed in $10 \%$ formalin. Staining with Masson's trichrome was performed according to standard protocols (16). Decidual vessels were identified (4-5/ placenta), and a blinded operator used ImageJ software (NIH) to measure the luminal area.

Radiotelemetry. Nonpregnant BPH/5 and C57 female mice underwent carotid implantation of telemetry devices (Data Sciences International) according to previously published methods (15). Briefly, female mice were anesthetized for placement of the telemeter in the thoracic aorta via the left carotid artery, and the transmitter body was placed in the lateral s.c. space. Mice were allowed to recover for 10 days, and then baseline MAP was recorded for 4 days before the introduction of a male. The day of vaginal plug was noted, and recordings were made from that day until delivery and for approximately 5 days postpartum.

Statistics. All data are expressed as the mean \pm SEM. Multiple comparisons were made using a 1- or 2-way ANOVA, where appropriate, followed by post-hoc analysis. A 1- or 2-tailed Student's $t$ test was used for all other analyses. Statistical significance was defined as a $P$ value of less than 0.05 .

Study approval. All animal procedures were approved by the Cornell University Animal Care and Use Committee. Care of the mice met the standards set forth by the NIH guidelines on the care and use of animals, USDA regulations, and the American Veterinary Medical Association (AVMA) Panel on Euthanasia.

\section{Author contributions}

JLS, JC, AKW, HEL, SKD, and RLD conceived and designed the research. JLS, JC, AKW, AB, CYH, HEL, CEI, SDB, and SES performed the experiments. JLS, JC, AKW, HEL, CYH, CEI, and SES analyzed the data. JLS, JC, CYH, HEL, SKD, and RLD interpreted the experiment results. JLS prepared the figures and drafted the manuscript. JLS, JC, SKD, and RLD edited and revised the manuscript. JLS and RLD approved the final version of the manuscript.

\section{Acknowledgments}

The authors wish to thank Laurie Lacko and Yi Zhou for technical assistance; Heidi Stuhlman and Colin Young for important discussions; and Jen Musa for assistance with editing the manuscript. This work was supported by grants from the Qatar National Research Fund (NPRP09-1099-279, NIH HL063887, and HL084207, to R.L. Davisson); the March of Dimes (3-FY12-127, 33-FY13-543, to S.K. Dey); and the NIH 
(HD068524 and CA7783, to S.K. Dey). J.L. Sones and A.K. Woods were supported by American Heart Association fellowships (12POST11250010 and 09PRE2120035, respectively) and J. Cha by an individual predoctoral National Research Service Award (NIH F30AG040858).

Address correspondence to: Robin L. Davisson, T9-014C Veterinary Research Tower, Cornell University, Ithaca, New York 14853-6401, USA. Phone: 607.253.3537; E-mail: robin.davisson@cornell.edu.

1. Cheng MH, Wang PH. Placentation abnormalities in the pathophysiology of preeclampsia. Expert Rev Mol Diagn. 2009;9(1):37-49.

2. Soto E, et al. Late-onset preeclampsia is associated with an imbalance of angiogenic and anti-angiogenic factors in patients with and without placental lesions consistent with maternal underperfusion. J Matern Fetal Neonatal Med. 2012;25(5):498-507.

3. Torry DS, Hinrichs M, Torry RJ. Determinants of placental vascularity. Am J Reprod Immunol. 2004;51(4):257-268.

4. Ramathal CY, Bagchi IC, Taylor RN, Bagchi MK. Endometrial decidualization: of mice and men. Semin Reprod Med. 2010;28(1):17-26

5. Cha J, Sun X, Dey SK. Mechanisms of implantation: strategies for successful pregnancy. Nat Med. 2012;18(12):1754-1767.

6. Das SK, Dey SK. Embryo-Uterine Interactions during Implantation: Potential Sites of Interference by Environmental Toxins. 2nd ed. Millbrae, California, USA: Elsevier Science Ltd; 2010.

7. Dey SK, et al. Molecular cues to implantation. Endocr Rev. 2004;25(3):341-373.

8. Lim H, et al. Cyclo-oxygenase-2-derived prostacyclin mediates embryo implantation in the mouse via PPARdelta. Genes Dev. 1999;13(12):1561-1574.

9. Song H, Lim H, Das SK, Paria BC, Dey SK. Dysregulation of EGF family of growth factors and COX-2 in the uterus during the preattachment and attachment reactions of the blastocyst with the luminal epithelium correlates with implantation failure in LIF-deficient mice. Mol Endocrinol. 2000;14(8):1147-1161.

10. Matsumoto $\mathrm{H}$, et al. Cyclooxygenase-2 differentially directs uterine angiogenesis during implantation in mice. J Biol Chem. 2002;277(32):29260-29267.

11. Erlebacher A. Immunology of the maternal-fetal interface. Annu Rev Immunol. 2013;31:387-411.

12. Burke SD, et al. Uterine NK cells, spiral artery modification and the regulation of blood pressure during mouse pregnancy. $A m J$ Reprod Immunol. 2010;63(6):472-481.

13. Herington JL, Bany BM. Effect of the conceptus on uterine natural killer cell numbers and function in the mouse uterus during decidualization. Biol Reprod. 2007;76(4):579-588.

14. Murphy SP, Tayade C, Ashkar AA, Hatta K, Zhang J, Croy BA. Interferon $\gamma$ in successful pregnancies. Biol Reprod. 2009;80(5):848-859.

15. Davisson RL, et al. Discovery of a spontaneous genetic mouse model of preeclampsia. Hypertension. 2002;39(2 pt 2):337-342.

16. Dokras A, et al. Severe feto-placental abnormalities precede the onset of hypertension and proteinuria in a mouse model of preeclampsia. Biol Reprod. 2006;75(6):899-907.

17. Hoffmann DS, et al. Chronic tempol prevents hypertension, proteinuria, and poor feto-placental outcomes in BPH/5 mouse model of preeclampsia. Hypertension. 2008;51(4):1058-1065.

18. Woods AK, et al. Adenoviral delivery of VEGF121 early in pregnancy prevents spontaneous development of preeclampsia in BPH/5 mice. Hypertension. 2011;57(1):94-102.

19. Bujold E, Roberge S, Nicolaides KH. Low dose aspirin for prevention of adverse outcomes related to abnormal placentation. Prenat Diagn. 2014;34(7):642-648.

20. Cross JC. The genetics of pre-eclampsia: a feto-placental or maternal problem? Clin Genet. 2003;64(2):96-103

21. Psychoyos A. Hormonal control of ovoimplantation. Vitam Horm. 1973;31:201-256.

22. Ye X, et al. LPA3-mediated lysophosphatidic acid signalling in embryo implantation and spacing. Nature. 2005;435(7038):104108

23. Song H, et al. Cytosolic phospholipase A2 $\alpha$ is crucial [correction of A2 $\alpha$ deficiency is crucial] for 'on-time' embryo implantation that directs subsequent development. Development. 2002;129(12):2879-2889.

24. Schlager G, Sides J. Characterization of hypertensive and hypotensive inbred strains of mice. Lab Anim Sci. 1997;47(3):288-292.

25. Wang H, Dey SK. Roadmap to embryo implantation: clues from mouse models. Nat Rev Genet. 2006;7(3):185-199.

26. Franco HL, et al. WNT4 is a key regulator of normal postnatal uterine development and progesterone signaling during embryo implantation and decidualization in the mouse. FASEB J. 2011;25(4):1176-1187.

27. Finn CA, Martin L. Endocrine control of the timing of endometrial sensitivity to a decidual stimulus. Biol Reprod. 1972;7(1):8286.

28. Lim H, et al. Multiple female reproductive failures in cyclooxygenase 2-deficient mice. Cell. 1997;91(2):197-208

29. Paria BC, et al. Cellular and molecular responses of the uterus to embryo implantation can be elicited by locally applied growth factors. Proc Natl Acad Sci U S A. 2001;98(3):1047-1052.

30. Lee KY, et al. Bmp2 is critical for the murine uterine decidual response. Mol Cell Biol. 2007;27(15):5468-5478.

31. Yadi H, Burke S, Madeja Z, Hemberger M, Moffett A, Colucci F. Unique receptor repertoire in mouse uterine NK cells. $J$ Immunol. 2008;181(9):6140-6147.

32. Chen Z, et al. DBA-lectin reactivity defines mouse uterine natural killer cell subsets with biased gene expression. Biol Reprod. 2012;87(4):81.

33. Croy BA, Chantakru S, Esadeg S, Ashkar AA, Wei Q. Decidual natural killer cells: key regulators of placental development (a review). J Reprod Immunol. 2002;57(1-2):151-168.

34. Elpek KG, Rubinstein MP, Bellemare-Pelletier A, Goldrath AW, Turley SJ. Mature natural killer cells with phenotypic and 
functional alterations accumulate upon sustained stimulation with IL-15/IL-15Ralpha complexes. Proc Natl Acad Sci U S A. 2010;107(50):21647-21652.

35. Sookvanichsilp N, Pulbutr P. Anti-implantation effects of indomethacin and celecoxib in rats. Contraception. 2002;65(5):373-378.

36. Shishodia S, Koul D, Aggarwal BB. Cyclooxygenase (COX)-2 inhibitor celecoxib abrogates TNF-induced NF-kB activation through inhibition of activation of I $\mathrm{KB} \alpha$ kinase and Akt in human non-small cell lung carcinoma: correlation with suppression of COX-2 synthesis. J Immunol. 2004;173(3):2011-2022.

37. Urato AC, Norwitz ER. A guide towards pre-pregnancy management of defective implantation and placentation. Best Pract Res Clin Obstet Gynaecol. 2011;25(3):367-387.

38. Maynard SE, et al. Excess placental soluble fms-like tyrosine kinase 1 (sFlt1) may contribute to endothelial dysfunction, hypertension, and proteinuria in preeclampsia. J Clin Invest. 2003;111(5):649-658.

39. Nash P, et al. Placental dysfunction in Suramin-treated rats - a new model for pre-eclampsia. Placenta. 2005;26(5):410-418

40. Wilcox AJ, Baird DD, Weinberg CR. Time of implantation of the conceptus and loss of pregnancy. N Engl J Med. 1999;340(23):1796-1799.

41. Daikoku T, et al. Conditional deletion of Msx homeobox genes in the uterus inhibits blastocyst implantation by altering uterine receptivity. Dev Cell. 2011;21(6):1014-1025.

42. Stewart CL, et al. Blastocyst implantation depends on maternal expression of leukaemia inhibitory factor. Nature. 1992;359(6390):76-79.

43. Song H, Lim H. Evidence for heterodimeric association of leukemia inhibitory factor (LIF) receptor and gp130 in the mouse uterus for LIF signaling during blastocyst implantation. Reproduction. 2006;131(2):341-349.

44. Li M, Yee D, Magnuson TR, Smithies O, Caron KM. Reduced maternal expression of adrenomedullin disrupts fertility, placentation, and fetal growth in mice. J Clin Invest. 2006;116(10):2653-2662.

45. Remuzzi G, et al. Reduced umbilical and placental vascular prostacyclin in severe pre-eclampsia. Prostaglandins. 1980;20(1):105-110.

46. Hanna J, et al. Decidual NK cells regulate key developmental processes at the human fetal-maternal interface. Nat Med. 2006;12(9):1065-1074

47. Williams PJ, Bulmer JN, Searle RF, Innes BA, Robson SC. Altered decidual leucocyte populations in the placental bed in pre-eclampsia and foetal growth restriction: a comparison with late normal pregnancy. Reproduction. 2009;138(1):177-184

48. Barber EM, Pollard JW. The uterine NK cell population requires IL-15 but these cells are not required for pregnancy nor the resolution of a Listeria monocytogenes infection. J Immunol. 2003;171(1):37-46.

49. Toth B, et al. Placental interleukin-15 expression in recurrent miscarriage. Am J Reprod Immunol. 2010;64(6):402-410.

50. Hu W, Wang H, Wang Z, Huang H, Dong M. Elevated serum levels of interleukin-15 and interleukin-16 in preeclampsia. $J$ Reprod Immunol. 2007;73(2):166-171.

51. Bachmayer N, Rafik Hamad R, Liszka L, Bremme K, Sverremark-Ekstrom E. Aberrant uterine natural killer (NK)-cell expression and altered placental and serum levels of the NK-cell promoting cytokine interleukin-12 in pre-eclampsia. Am J Reprod Immunol. 2006;56(5-6):292-301.

52. Majed BH, Khalil RA. Molecular mechanisms regulating the vascular prostacyclin pathways and their adaptation during pregnancy and in the newborn. Pharmacol Rev. 2012;64(3):540-582.

53. Klockenbusch W, Goecke TW, Krussel JS, Tutschek BA, Crombach G, Schror K. Prostacyclin deficiency and reduced fetoplacental blood flow in pregnancy-induced hypertension and preeclampsia. Gynecol Obstet Invest. 2000;50(2):103-107.

54. Min SY, et al. Increase of cyclooxygenase-2 expression by interleukin 15 in rheumatoid synoviocytes. J Rheumatol. 2004;31(5):875-883

55. Sykes L, MacIntyre DA, Teoh TG, Bennett PR. Anti-inflammatory prostaglandins for the prevention of preterm labour. Reproduction. 2014;148(2):R29-40.

56. Hirota Y, Daikoku T, Tranguch S, Xie H, Bradshaw HB, Dey SK. Uterine-specific p53 deficiency confers premature uterine senescence and promotes preterm birth in mice. J Clin Invest. 2010;120(3):803-815

57. Cha J, et al. Combinatory approaches prevent preterm birth profoundly exacerbated by gene-environment interactions. J Clin Invest. 2013;123(9):4063-4075.

58. Van der Auwera I, D'Hooghe T. Superovulation of female mice delays embryonic and fetal development. Hum Reprod. $2001 ; 16(6): 1237-1243$

59. Slevin JC, et al. High resolution ultrasound-guided microinjection for interventional studies of early embryonic and placental development in vivo in mice. BMC Dev Biol. 2006;6:10.

60. Young $\mathrm{CN}$, et al. ER stress in the brain subfornical organ mediates angiotensin-dependent hypertension. J Clin Invest. 2012;122(11):3960-3964.

61. Pakrasi PL, Jain AK. Cyclooxygenase-2 derived PGE2 and PGI2 play an important role via EP2 and PPARdelta receptors in early steps of oil induced decidualization in mice. Placenta. 2008;29(6):523-530.

62. Salker MS, et al. Deregulation of the serum- and glucocorticoid-inducible kinase SGK1 in the endometrium causes reproductive failure. Nat Med. 2011;17(11):1509-1513. 